\title{
DECONVOLVING STRAIN MAPS OBTAINED WITH THE GRID METHOD
}

\author{
M. Grédiac ${ }^{1}$, F. Sur ${ }^{2}$, C. Badulescu ${ }^{3}$, J-D. Mathias ${ }^{4}$ \\ ${ }^{1}$ Clermont Université, Université Blaise Pascal, Institut Pascal, UMR CNRS 6602 \\ BP 10448, 63000 Clermont-Ferrand, France \\ ${ }^{2}$ Laboratoire Lorrain de Recherche en Informatique et ses Applications, UMR CNRS 7503 \\ Université de Lorraine, CNRS, INRIA projet Magrit, Campus Scientifique, BP 239 \\ 54506 Vandoeuvre-lès-Nancy Cedex, France \\ ${ }^{3}$ Laboratoire Brestois de Mécanique et des Systèmes \\ ENSTA Bretagne, 2 rue François Verny, 29806 Brest Cedex 9, France \\ ${ }^{4}$ IRSTEA, Laboratoire d'Ingénierie pour les Systèmes Complexes \\ Campus universitaire des Cézeaux, 24 avenue des Landais, BP50085, 63172 Aubière Cedex, France
}

\begin{abstract}
The use of various deconvolution techniques to enhance strain maps obtained with the grid method is addressed in this study. Since phase derivative maps obtained with this measurement technique can be approximated by their actual counterparts convolved by the envelope of the kernel used to extract phases and phase derivatives, non-blind restoration techniques can be used to perform deconvolution. Six deconvolution techniques are compared here in order to restore a synthetic phase derivative map. Obtained results are analyzed and discussed.
\end{abstract}

Keywords: deconvolution, displacement, grid method, strain measurement, metrological performance

\section{INTRODUCTION}

Full-field measurement techniques are now wide spread in the experimental mechanics community. One of the reasons is their ability to measure heterogeneous states of strain and to detect localized events that often occur in specimens under test. Assessing the metrological performances of full-field measurement techniques remains an open issue. Recent papers published in this field mainly deal with digital image correlation [1-2] for instance. One of the problems is that these performances depend on various parameters whose individual contribution is generally difficult to estimate [3].

Another problem is the fact that metrological performances depend on two conflicting concepts: resolution and spatial resolution. The first one is defined here by the smallest strain or displacement that can be detected, the second one by the smallest distance between independent measurements. It is well known that the better resolution, the worst spatial resolution. In addition, displacement is generally the physical quantity which is provided by many techniques but strain is the physical quantity involved in constitutive equations of engineering materials, so it often needs to be calculated. Since displacement maps are generally noisy, they are smoothed before differentiation which is performed to obtain strain components [4]. In conclusion, the number of parameters influencing the metrological performance is significant. This leads to confused ideas on 
the actual metrological performances of full-field measurement techniques, especially when strain components are considered instead of displacements.

This paper is devoted to the problem of the actual strain determination in case of localized phenomena which occur for instance when heterogeneous materials are tested. It is well known that with any measurement technique, smoothing performed to limit the impact of noise impairs both the size of the details in strain maps and the strain amplitude in these zones: details are generally enlarged due to blurring and the amplitude of the identified strain value is lower than the actual one.

The measurement method employed here is the grid method, which relies on a regular bi-directional marking of the surface under investigation. Images of this grid are taken while testing the specimens. These images are then processed to retrieve the in-plane displacement and strain components. This surface preparation is in general more difficult to obtain than a speckle, which is the typical marking used for digital image correlation. The big advantage of grids is however that a regular marking is much easier to duplicate or to control than a random one. In addition, a regular marking makes it possible to use powerful image processing techniques based on the Fourier transform for which a broad literature is available [5].

It has been recently shown that displacement and strain maps obtained with the grid method can be approximated with their actual counterparts convolved by the envelope used to extract the phase and phase derivatives from the grid images by windowed Fourier transform [6]. The idea is therefore to use deconvolution technique in order to restore displacement and strain fields and obtain actual distributions of these quantities. This paper is solely devoted to phase derivative. Indeed the change of this quantity during a test is directly proportional to strain maps. Finding phase derivatives is more critical than finding phases only. The reason is that spatial differentiation requires a smoothing stage to reduce the impact of noise. This procedure induces more severe convolution for phase derivatives than for phases.

Deconvolution techniques have only seldom been employed in the field of mechanics [7-8]. Concerning displacement and strain measurement, one of the main reasons is certainly that the "classic" procedure to find strain maps, which consists in finding first displacement maps, smoothing them and differentiate the obtained result, involves several non-linear steps and numerous parameters. Consequently, the retrieved displacement and strain components do not appear as the convolution of the corresponding actual quantities in this case.

In this context, the aim of this paper is to show that deconvolution can be applied to restore strain maps obtained with the grid method. The main steps of the grid method are first recalled. The basics of deconvolution are then described along with the characteristics of various deconvolution algorithms. The performance of these algorithms is then briefly illustrated through the deconvolution of a synthetic phase derivative map.

\section{THE PHASE AND PHASE DERIVATIVES PROVIDED BY THE GRID METHOD AS THE CONVOLUTION OF THEIR ACTUAL COUNTERPARTS}

The grid method consists first in depositing a crossed grid on the surface under investigation in order to track the change in the geometry of the grid while loading increases, and to deduce the $2 \mathrm{D}$ strain fields from these images. The typical value of the pitch (denoted p) is $0.2 \mathrm{~mm}$. The procedure for depositing the grids is given in [9].

Processing grid images consists first in extracting the phases along directions $\mathrm{x}$ and $\mathrm{y}$ both in the reference and in the current images. The displacement is obtained from the phase changes along both directions using the following equation:

$$
\tilde{u}_{i}=-\frac{p}{2 \pi} \Delta \widetilde{\Phi}_{i}, i=x, y
$$

for the displacements and

$$
\widetilde{\varepsilon}_{i j}=-\frac{p}{4 \pi} \Delta\left(\frac{\partial \widetilde{\Phi}_{i}}{\partial x_{j}}+\frac{\partial \widetilde{\Phi}_{j}}{\partial x_{i}}\right), i, j=x, y
$$


for the strain components. It has been recently demonstrated in [6] that each of the quantities $\tilde{X}$ defined above $\left(\tilde{X}=\widetilde{\Phi}_{i}, \frac{\partial \widetilde{\Phi}_{i}}{\partial x_{j}}, \tilde{u}_{i}, \widetilde{\varepsilon}_{i j}, i, j=x, y\right)$ is nearly equal to the convolution of its actual counterpart $\mathrm{X}$ by the envelope of the kernel denoted g used in the windowed Fourier transform. Thus

$$
\tilde{X} \approx X^{*} g
$$

where the symbol * denotes convolution.

This property has two important consequences. The first one is that any sudden variation of the actual displacement (resp. strain) distribution is smoothed by the phase (resp. phase derivative) extraction procedure. Hence the maps obtained after applying this procedure are automatically blurred in zones featuring high strain gradients. The quantities provided by the grid image processing exhibit systematically a lower amplitude than the actual ones in these zones. In addition, the geometry of these details in the maps is also spread by convolution. The second consequence is that since such maps can be considered as maps of the actual quantity convolved by g, one can restore them and try to find the actual quantity by deconvolution.

The remark concerning deconvolution is especially true for strain maps. Indeed in this case, function g generally defines a wide window in order to limit noise effect on the phase derivative. The classic route for finding the phase derivatives, namely finding first the displacements, smoothing them and differentiating the obtained result is slightly changed here by merging the first two steps. Thus enlarging the window when extracting the phases reduces noise effect, so no further smoothing is necessary prior to differentiation to obtain phase derivatives. The main advantage of this procedure is that the link between phase derivatives and grid image is straightforward, thus deconvolution can be performed.

\section{PHASE AND PHASE DERIVATIVES RESTORATION BY DECONVOLUTION}

Deconvolution of noisy maps is an ill-posed inverse problem which is prone to noise amplification and artefacts such as the so-called ringing artifact [5]. Solving this type of problem can only be efficiently performed by using suitable strategies which generally rely on various assumptions which enable us to add some a priori information, and therefore to reduce the abovementioned problems.

Various deconvolution techniques have been tested in this study in order to observe and quantify their ability to restore correctly strain maps. These techniques are direct deconvolution, regularized (Tikhonov) deconvolution, Rirchardson-Lucy deconvolution and Wiener filtering, with two different variants concerning the last two. Full details concerning the application of these techniques to strain map restoration can be found in Ref. [10].

All these techniques have been tested in turn to deconvolve a synthetic phase derivative image containing a wide range of frequencies. The image of the synthetic $\frac{\partial \Phi_{y}}{\partial y}$ distribution is shown in Figure 1-a. As may be seen, the phase derivative amplitude is constant throughout the phase derivative map. It is equal to $10^{-3}$, which is a usual value for phase derivatives in deformed structures. The maximum frequency of the $\frac{\partial \Phi_{y}}{\partial y}$ distribution is located on the left-hand side of this figure. It is equal to half the frequency of the grid to avoid aliasing. All these quantities linearly evolve from the left- to the right-hand side, the maximum period being equal to 16 times that of the grid on the right-hand side. The idea is to observe at a glance both the effect of convolution due to phase derivative extraction and the ability of the deconvolution techniques listed above to restore the phase derivative distribution.

The corresponding phase derivative $\Phi_{\mathrm{y}}$ is merely obtained by integration and the other phase $\Phi_{\mathrm{x}}$ is assumed to be null. This information is used to modulate a grid whose pitch is equal to 5 pixels, and build a synthetic image for which the gray level is modelled as follows 

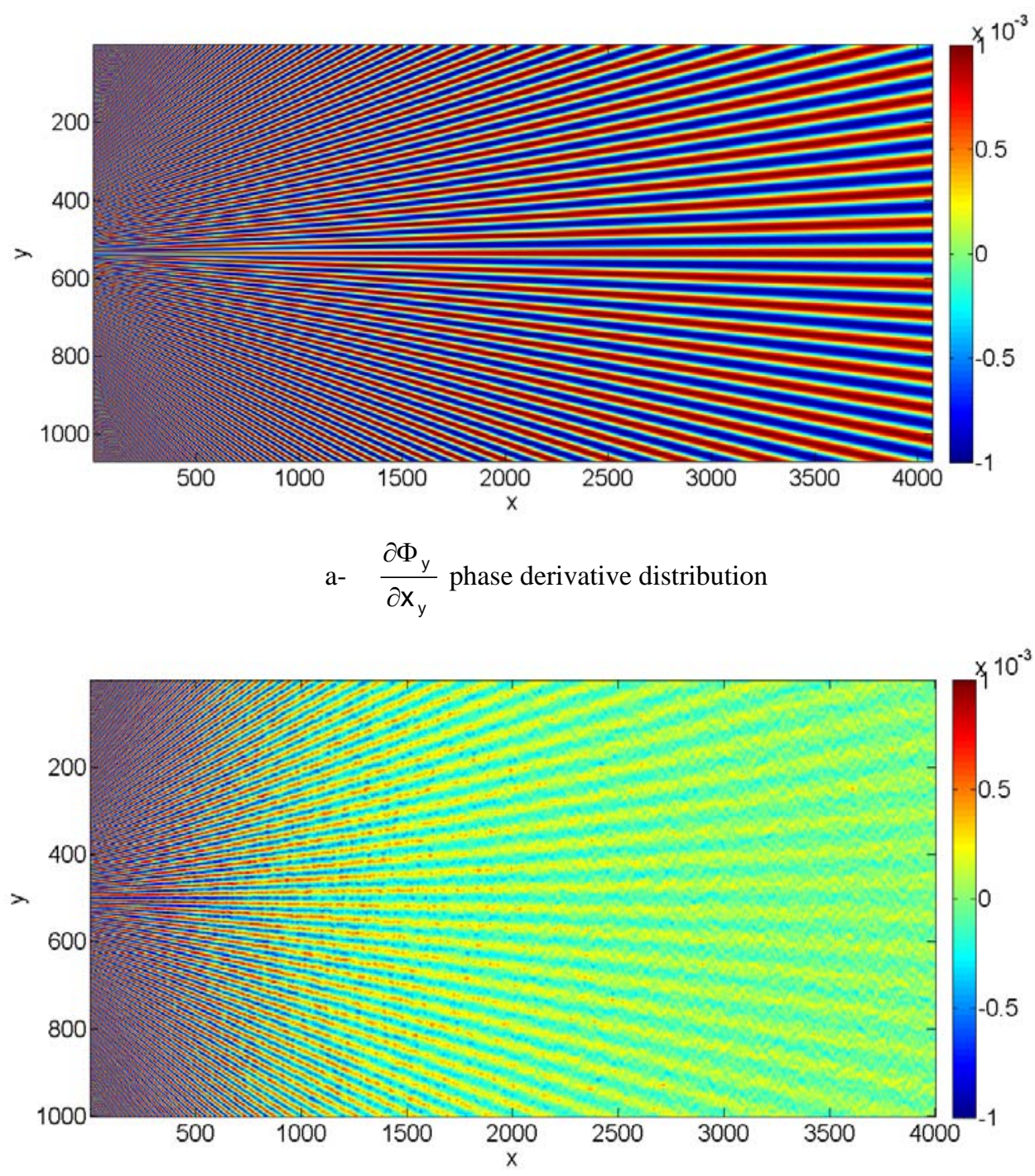

b- difference between reference and identified phase derivative distributions

Figure 1: synthetic distribution of the phase used to test various deconvolution techniques [10]

$$
s(x, y)=\frac{A}{2}\left[2+\sin ^{3}(2 \pi f x)+\sin ^{3}\left(2 \pi f y+\Phi_{y}\right)\right]
$$

where $A$ is the amplitude and $f$ the spatial grid frequency. Cubic sine functions are used here instead of pure sine functions to illustrate the fact that only the first harmonic of the periodic encoding is used to retrieve the phase and its derivatives as mentioned in [6]. It must finally be pointed out that a Gaussian white noise with various amplitude values has been added to this synthetic grid image to assess the robustness of the procedure.

Figure 1-b shows the difference between the reference phase derivative and that obtained by applying the windowed Fourier transform. In this case, a noise (amplitude 5 pixels) has been added to the synthetic grid image modulated with the phase obtained by integrating the phase derivative distribution shown in Figure 1-a. A Gaussian window is employed in the windowed Fourier transform performed to retrieve the phase and phase derivatives. Its amplitude is equal to 5 pixels, which is a typical value employed in practice. It can be seen that the higher the frequency, the greater the error between identified and reference phase derivatives. 


\section{TYPICAL RESULTS}

Typical examples of phase derivative maps restored with some deconvolution techniques are shown in Figure 2. It can be seen that regularized deconvolution is unstable whereas it can be checked that this technique is able to restore very efficiently phase derivative maps when no noise is added (results not shown here). This illustrates the fact that deconvolution is an illposed problem which must be solved with care.

Figure 2-b shows that the Richardson-Lucy algorithm restores high frequencies but significant noise amplitude is visible. Since this algorithm only restores images containing positive quantities and since we deal here with phase derivatives which can be negative, deconvolution is performed on the exponential of the phase derivative map. The logarithm of the result is then taken to obtain the restored distribution.

The phase derivative map deconvolved with Wiener filtering in shown in Figure 2-c. Comparing this map with that shown in Figure 1-b shows that this is a good compromise between high frequencies restoration and noise level in the phase derivative deconvolved map. A more refined analysis of the results obtained with the latter technique shows that Wiener filtering outperforms the Richardson-Lucy algorithm if the noise in the maps to be deconvolved is correctly characterized [10]. It has indeed been demonstrated in [6] that this noise is spatially correlated, thus deconvolution benefits from taking its autocorrelation function into account.

\section{CONCLUSION}

Various deconvolution techniques were used to restore strain fields obtained with the grid method. A synthetic example is used to assess the performance of these deconvolution techniques. The influence of noise on the quality of the deconvolved maps is a major issue since some techniques are totally unstable when noise is taken into account whereas they give good results without noise in the grid images. Numerical simulations show that the Richardson-Lucy algorithm and Wiener filtering give good results in terms of compromise between noise level in the restored maps and ability to restore rather high frequencies.

\section{REFERENCES}

[1] M. Bornert, F. Brémand, P. Doumalin, J.-C. Dupré, M. Fazzini, M. Grédiac, F. Hild, S. Mistou, J. Molimard, J.-J. Orteu, L. Robert, Y. Surrel, P. Vacher, and B. Wattrisse. Assessment of digital image correlation measurement errors: methodology and results. Experimental Mechanics, 49(3):353-370, 2009.

[2] P. Lava, S. Cooreman, S. Coppieters, M. De Strycker, and D. Debruyne. Assessment of measuring errors in DIC using deformation fields generated by plastic FEA. Optics and Lasers in Engineering, 47:747-753, 2009

[3] Y. Q. Wang, M.A. Sutton, H.A. Bruck, and H.W. Schreier. Quantitative error assessment in pattern matching: effects of intensity pattern noise, interpolation, strain and image contrast on motion measurements. Strain, 45:160-178, 2009

[4] L.B. Meng, G.C. Jin, and X.F. Yao. Application of iteration and finite element smoothing technique for displacement and strain measurement of digital speckle correlation. Optics and Lasers in Engineering, 45(1):57-63, 2007

[5] R.C. Gonzalez and R.E. Woods. Digital Image Processing (3rd Edition). Prentice-Hall, 2006

[6] F. Sur and M. Grédiac. Towards deconvolution to enhance the grid method for in-plane strain measurement. 2012. Submitted

[7] A. M. Maas and P. A. A. M. Somers. Two-dimensional deconvolution applied to phase-stepped shearography. Optics and Lasers in Engineering, 26:1594-1602, 2006

[8] B. Dhanasekar and B.Ramamoorthy. Restoration of blurred images for surface roughness evaluation using machine vision. Tribology International, 43:268-276, 2010

[9] J.L. Piro and M. Grédiac, Producing and transferring low-spatial-frequency grids for measuring displacement fields with moiré and grid methods, Experimental Techniques, 28(4), 23-26, 2004

[10] M. Grédiac, F. Sur, C. Badulescu, and J.-D. Mathias. Using deconvolution to improve the metrological performance of the grid method. Optics and Lasers in Engineering, in press, 2013 


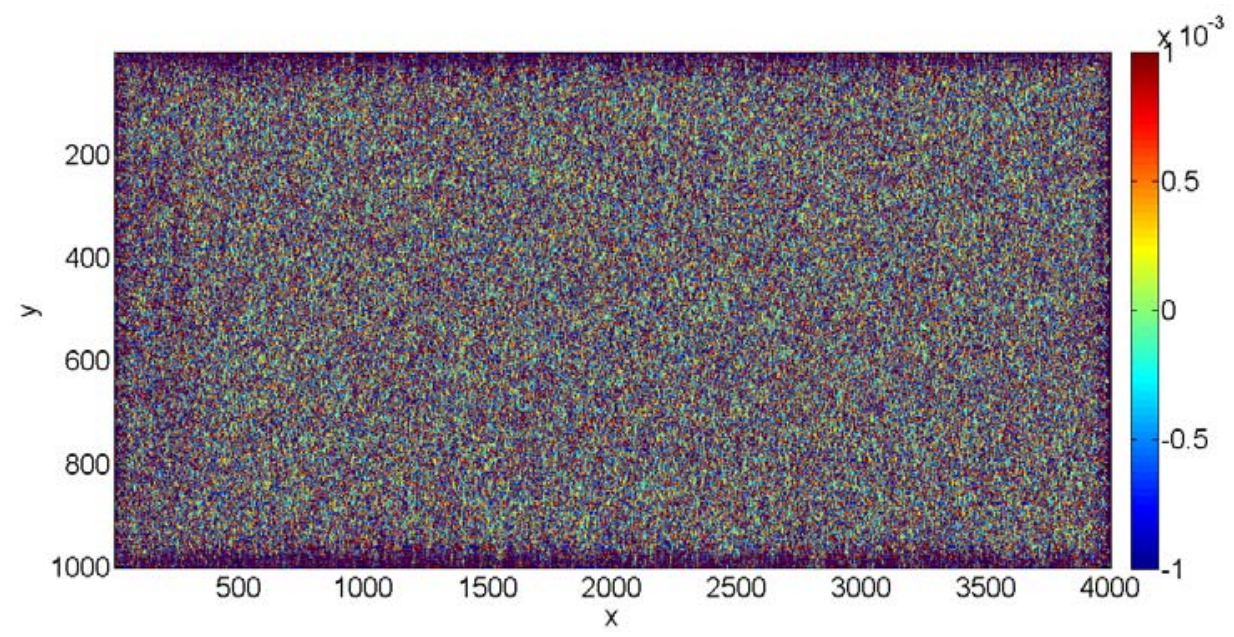

a- Regularized deconvolution

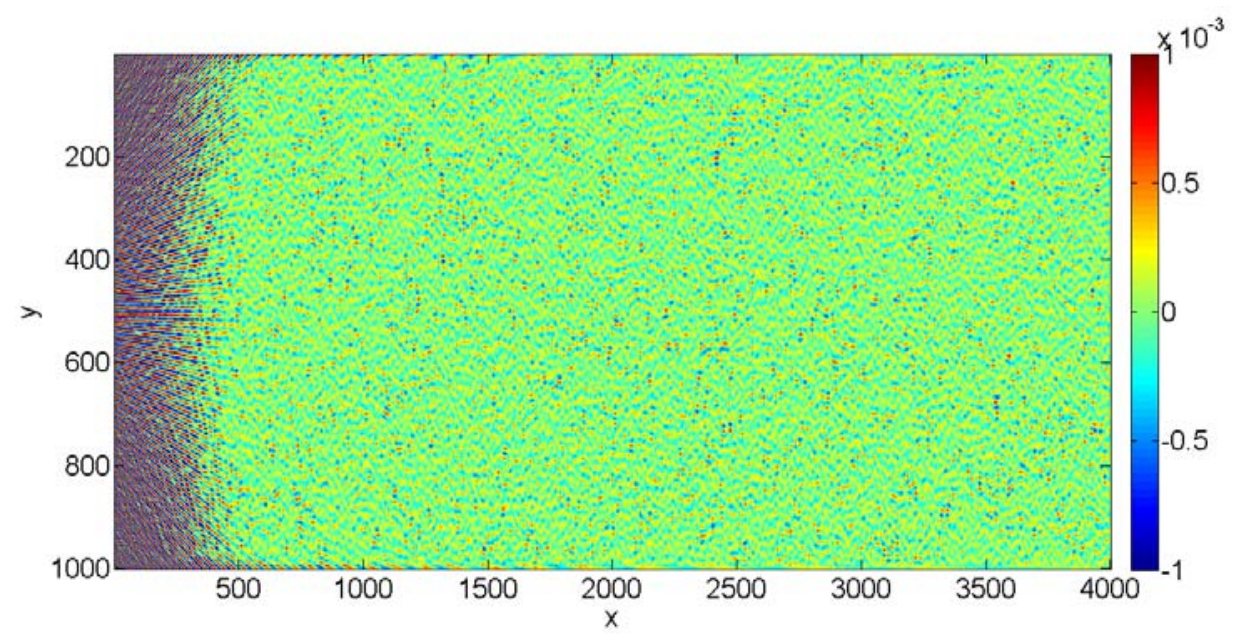

b- Richardson Lucy algorithm

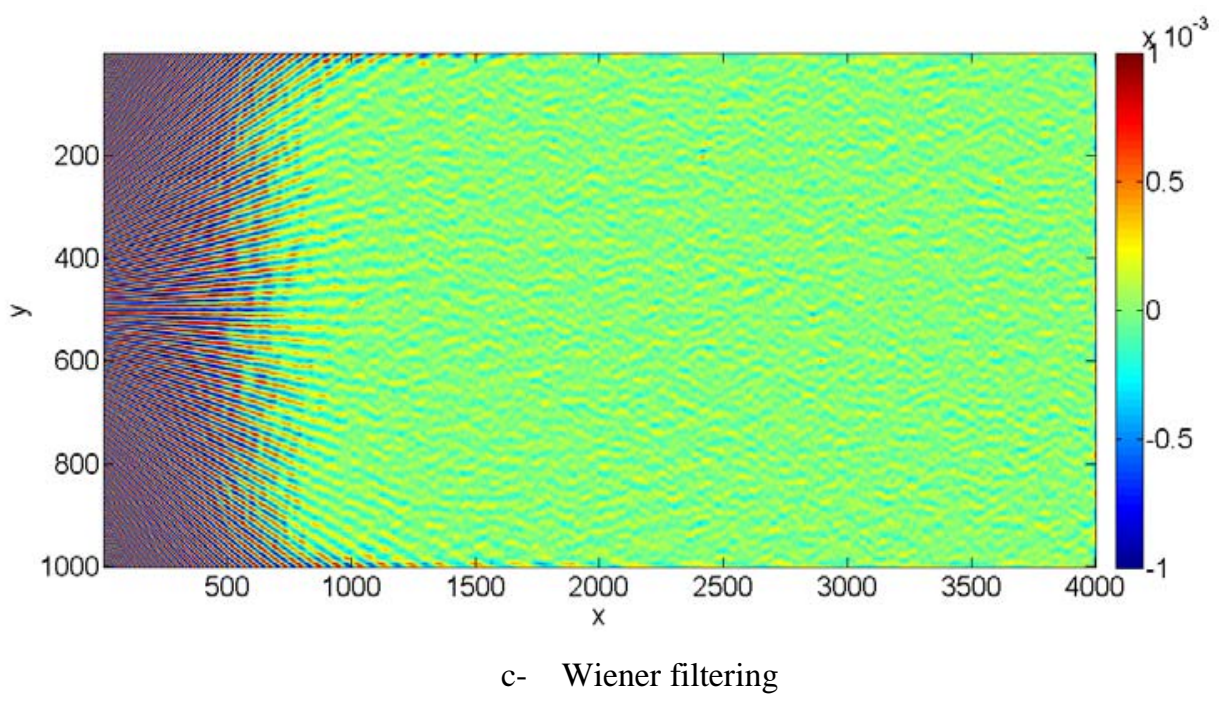

Figure 2: example of phase derivative maps obtained after deconvolution with various techniques [10] 